\title{
Efectos y perspectivas económicas de la presencia del COVID-19 en la región Tacna
}

\author{
Effects and economic perspectives of the presence of COVID-19 in the Tacna region
}

\author{
Edwin Ismael Palza Chambe \\ Universidad Nacional Jorge Basadre Grohmann, \\ Tacna-Perú. \\ ORCID: 0000-0001-6411-2907 \\ epalzach@unjbg.edu.pe
}

\section{RESUMEN}

La declaración de la pandemia mundial por la presencia del denominado COVID-19 ha generado una serie de cambios e impactos en el desarrollo normal de los diversos espacios territoriales, entre ellos el de la región Tacna. Es así que desde la aparición del primer caso en el Perú (un 06 de marzo del 2020), se implementaron una serie de medidas para mitigar el nivel de contagio en el país, entre ellos: el confinamiento obligatorio y el cierre de fronteras. La puesta en alerta y, en ciertos momentos, el colapso del sistema de salud determinaron impactos en la morbilidad y mortalidad de la región, pero también se suscitaron otros efectos ya vinculados directamente con lo económico y productivo. Los cuales son evaluados en términos nacionales, pero no inclinándose en una revisión de los comportamientos específicos regionales. El trabajo formulado identifica que la captación de tributos internos para el período de enero a agosto del 2020 se ha reducido en 22.98 $\%$ (pero $24.21 \%$ en términos reales), lo cual en base al análisis formulado implicaría una contracción en el PBI para el mismo año del orden del $22.44 \%$ y un incremento en la tasa de desempleo de $32.06 \%$.

Palabras clave: Desempleo, gobierno, ingresos, ley de Okun, producción, tributos.

\begin{abstract}
The declaration of the global pandemic due to the presence of the so-called COVID-19 has generated a series of changes and impacts on the normal development of the different territorial spaces, among them the Tacna region. Since the first case appeared in Peru (on March 6, 2020), a series of steps have been taken to mitigate the level of infection in the country, including compulsory confinement and border closures. The warning and, at certain times, the collapse of the health system had an impact on morbidity and mortality in the region, but there were also other effects directly related to the economy and production. These are evaluated in national terms, but not in terms of a review of specific regional behaviors. The analysis identifies that the collection of internal taxes for the period from January to August 2020 has been reduced by $22.98 \%$ (but $24.21 \%$ in real terms), which, based on the analysis, would imply a contraction in the GDP for the same year in the order of $22.44 \%$ and an increase in the unemployment rate of $32.06 \%$.
\end{abstract}

Keywords: Unemployment, government, income, Okun's law, production, taxes.

\section{INTRODUCCIÓN}

El término COVID-19 (derivado de las siglas en inglés para coronavirus disease 2019), tan reiterado en estos meses, es la denominación asignada a una enfermedad causada por el virus SARS-CoV-2; la cual fue declarada como pandemia por la Organización Mundial de la Salud el 11 de marzo del 2020. Este hecho así como la presencia del primer caso en el Perú suscitaron la emisión del Decreto Supremo No 044-2020-PCM, mediante el cual se implementa el "Estado de Emergencia Nacional" y la implementación del aislamiento social obligatorio, en un período inicial de quince (15) días, lo cual fue consecutivamente ampliado y que para el caso de diversas regiones (como Tacna) se mantiene aún vigente a la fecha de elaboración de este documento.

DOI: https://doi.org/10.33326/27086062.2020.2.966

Presentado: 30/07/2020, Aceptado: 23/10/2020 
El primer caso registrado de la enfermedad en la región Tacna se suscitó el 29 de marzo y determinó un crecimiento de casos registrados diariamente, que fue paulatinamente acelerándose hasta alcanzar su cenit 153 días después (el 27 de agosto, cuando se reportan 457 casos), para iniciar a partir de allí un aparente repliegue de valores (como se aprecia en la Figura 01).

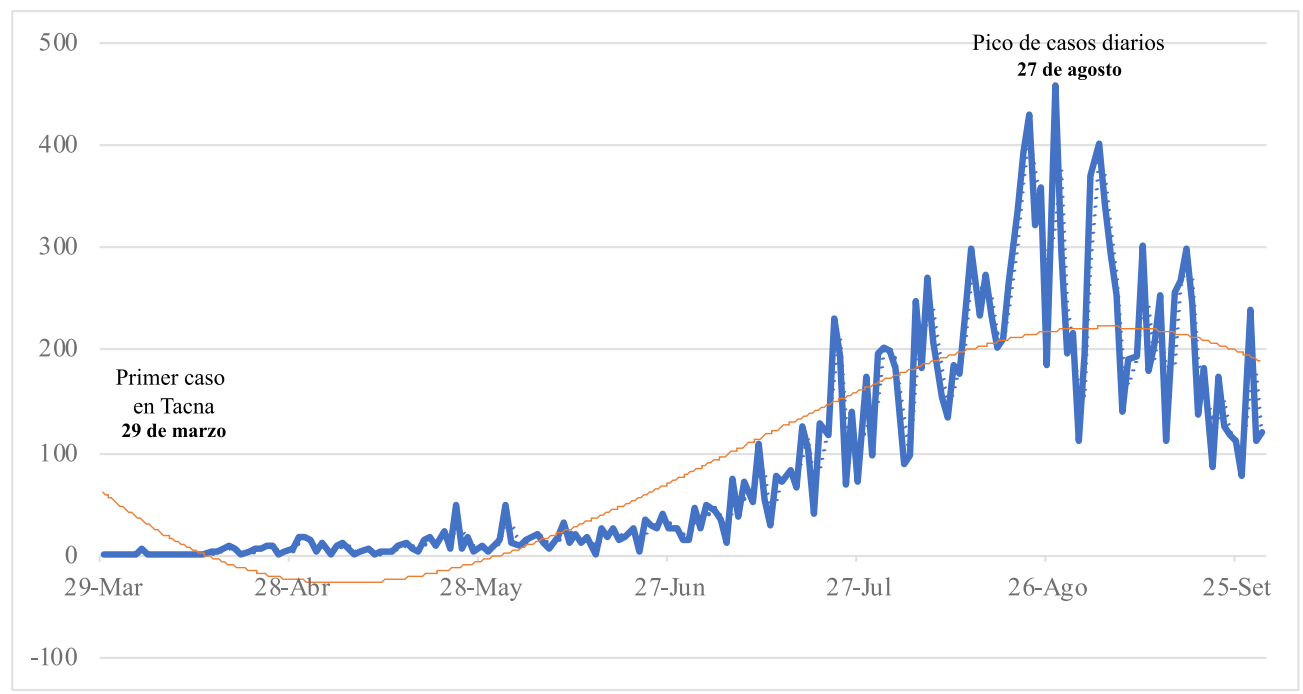

Figura 1. Región Tacna: Número de casos positivos diarios registrados por COVID-19

Fuente: Dirección Regional de Salud de Tacna (2020)

Aún resulta anticipado asumir que esta tendencia sea irreversible y que los casos de dicha enfermedad se minimicen decididamente, debido a que la experiencia de otros países y regiones (además de otras pandemias antes registradas en la historia de la humanidad) plantean la posibilidad de nuevas olas de contagio que sacudan nuevamente a la región y generen mayores impactos en las ya raleadas arcas de las familias y empresas de Tacna.

Uno de los principales resultados de la irrupción de la enfermedad es el crecimiento de la mortalidad, de un promedio de 3.71 fallecimientos/día registrado en el período 2017-2019 a 5.45 fallecimientos/día durante el año 2020 (considerando los datos hasta el 28 de setiembre). Si contemplamos los valores acumulados al mes de setiembre, el número de fallecidos en el presente año presenta valores de $34.98 \%$ superiores a los registrados en el 2019 (Sistema Informático Nacional de Defunciones, 2020). Evidentemente, esto puede verse explicado por los 562 fallecidos atribuidos al COVID-19 y los 83 fallecidos sospechosos de haber contraído el mal (Dirección Regional de Salud de Tacna, 2020).

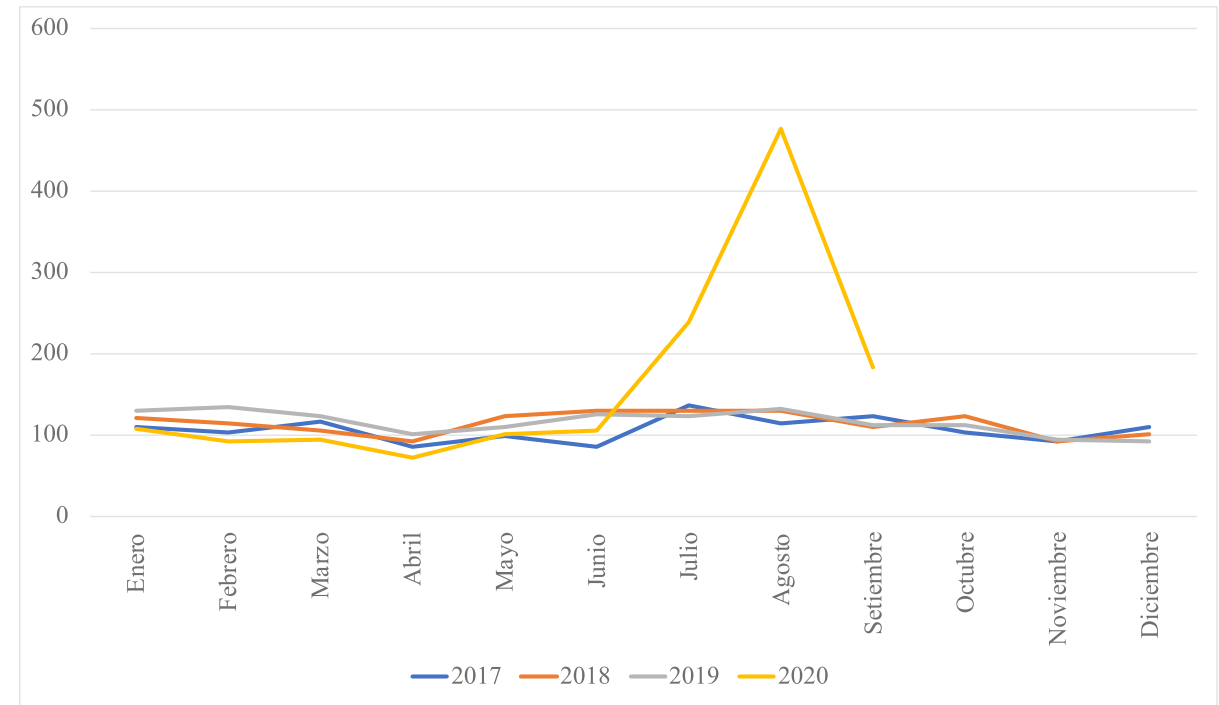

Figura 2. Región Tacna: Fallecimientos registrados por mes (enero 2017- setiembre 2020)

Fuente: Sistema Informático Nacional de Defunciones (2020) 
Sin embargo, la implementación de medidas como el confinamiento o el cierre de fronteras generan un impacto significativo en el desarrollo de las actividades económicas desplegadas en la región.

Cabe precisar que la frontera peruano-chilena, a pesar de ser una de las más pequeñas para nuestro país abarcando $169 \mathrm{~km}$ (lo que representa solo el $5.49 \%$ en referencia a la totalidad de la longitud limítrofe del país), es una frontera de reciente data y generada a partir de la firma del Tratado de Lima de 1929. La vinculación fundamentalmente se exalta entre las ciudades de Tacna y Arica, capitales de las regiones limítrofes, cuyo paso fronterizo constituye la segunda más dinámica en el cono sur de Sudamérica (solo superado por la denominada como "triple frontera", entre las ciudades de Ciudad del Este, Puerto Iguazú y Foz de Iguaçu).

Es así que la restricción en el desplazamiento de la población determina indudablemente una menor presencia de potenciales consumidores en los distintos establecimientos instalados en la región Tacna y, a partir de ello, una contracción del volumen de las ventas tanto en bienes y servicios.

Un acercamiento a la evaluación de estos impactos es el presentado en el "Informe de movilidad de las comunidades ante el COVID-19" (Google Inc., 2020), donde que en base a la evaluación de los portadores de equipos celulares con geoposicionador se puede percibir los cambios generados en sus desplazamientos habituales. En base a dichos datos se puede definir que las visitas a tiendas y espacios dedicados al ocio sufrió al inicio del "Estado Nacional de Emergencia" una contracción cercana al 80 \% del tráfico común, cifra similar a la registrada en los supermercados y farmacias de la región. Al inicio del mes de setiembre, la disminución de la movilidad se veía menguar al plantear una reducción cercana al $40 \%$ del volumen habitual de desplazamientos hacia ambos giros de negocios.
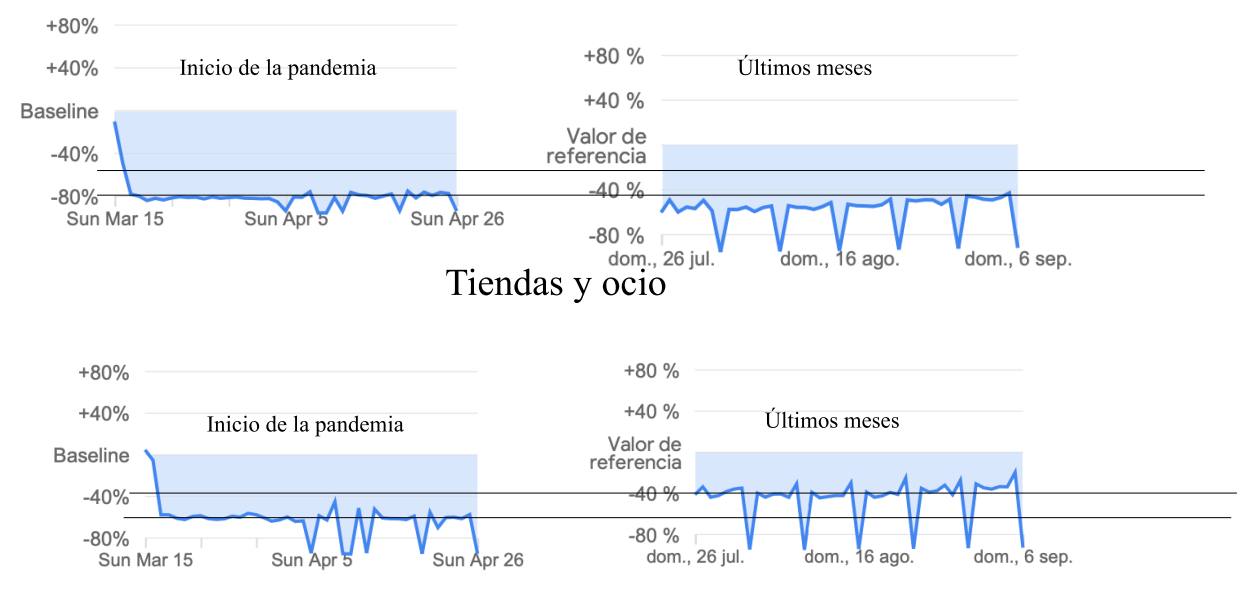

Supermercados y farmacias

Figura 3. Región Tacna: Movilidad de personas hacia tiendas, ocios, supermercados y farmacias (abril y setiembre 2020)

Fuente: Google Inc. (2020)

Un espacio fronterizo como es la ciudad de Tacna, capital de la región en análisis que a la par acoge a la mayor proporción de la población, es especialmente vulnerable a la ausencia de la llamada población "flotante", básicamente constituida por los visitantes y turistas chilenos (en esencia aquella proveniente de la ciudad de Arica).

Al respecto, Contreras, Tapia y Liberona (2017) indagan en los factores explicativos de esta alta movilidad desde Arica hacia Tacna, la cual es explicada por factores comerciales, turísticos, ocio, gastronómicos, entre otros; fundamentalmente orientado hacia un patrón de concentración y una economía de aglomeración de comercios, turismo y centros médicos en la ciudad de Tacna, que se localizan en torno a la Avenida Bolognesi y centro de la ciudad. Esto plantea, para Dilla y Álvarez (2018), una mejora en la dotación de servicios para esta ciudad (en relación a otras ciudades del país), aun cuando la mayor cantidad de visitantes de origen chileno tienen una corta estadía en la ciudad y básicamente son alentados por el menor costo de sus servicios (derivados a la par de la favorable diferencia cambiaria). Se destaca también la peculiar condición de esta urbe, con un enorme flujo de 
visitantes extranjeros orientados al consumo de servicios; y de nacionales, en camino hacia su mercado laboral. Ambos aspectos remecidos por las condiciones de cierre de frontera devenidos de la emergencia sanitaria que sacude al mundo en estos días.

Mucho se plantea por la alta susceptibilidad de Tacna y de su economía a las medidas que impliquen restricciones (en este caso explicadas por la presencia de la pandemia COVID-19) en el desplazamiento de su población y aquella que habitualmente acoge proveniente de Chile.

Subsiste, no obstante, la necesidad de tener mayor indicadores que nos permitan reflejar los impactos de esta emergencia sanitaria; fundamentalmente en lo referente a la producción y el desempleo, aspectos que la contabilidad nacional generalmente solo entrega en términos del país, lo que impide tener valores de espacios menores, los cuales resultan fundamentales para la toma de decisiones en estos ámbitos.

\section{MATERIAL Y MÉTODOS}

\section{Materiales}

Para el desarrollo del trabajo, se hizo uso de los recursos provistos por la Escuela Profesional de Ingeniería en Economía Agraria de la Facultad de Ciencias Agropecuarias de la Universidad Nacional Jorge Basadre Grohmann de Tacna, lo que básicamente implican la disposición de un equipo de cómputo y el software allí instalado.

\section{Tipo de investigación}

El tipo de investigación es aplicada, al generar conocimiento para su uso inmediato (en este caso en particular construir información económica agregada para la toma de decisiones de actores decisores).

\section{Enfoque de investigación}

El enfoque de la investigación es cuantitativa, al estar basada en la información numérica de las variables analizadas.

\section{Alcance de la investigación}

El estudio tiene un alcance descriptivo, el cual se encuentra delimitado del modo siguiente:

- Espacio: La jurisdicción de la región Tacna.

- Tiempo: Para la evaluación de las variables se contempla la disposición de datos correspondientes al período 2007-2016, con el propósito de inferir el comportamiento para el año 2020.

\section{Diseño de la investigación}

El diseño de investigación es de tipo no experimental seccional descriptivo.

\section{Operacionalización de variables}

Para determinar la necesidad de información se utilizó la siguiente matriz de operacionalización de variables:

Tabla 1. Matriz de operacionalización de variables de la investigación

\begin{tabular}{|c|c|c|}
\hline Variable & Dimensión & Indicadores \\
\hline \multirow[t]{2}{*}{$\begin{array}{l}\text { Efectos y perspectivas } \\
\text { económicas }\end{array}$} & Producción & $\begin{array}{l}\text { - Producto Bruto Interno (PBI) regional de } \\
\text { Tacna (expresado en términos constantes) } \\
\text { - Recaudación de tributos internos en la } \\
\text { región Tacna (expresado en términos } \\
\text { constantes) }\end{array}$ \\
\hline & Desempleo & $\begin{array}{l}\text { Tasa de desempleo (en porcentaje del } \\
\text { PEA) en la región Tacna }\end{array}$ \\
\hline
\end{tabular}




\section{Recopilación de datos}

Para formular el análisis, se obtuvieron datos básicamente de origen documental, los cuales se encuentran disponibles a través de las páginas web institucionales de las entidades como: Superintendencia Nacional de Administración Tributaria (SUNAT), Ministerio de Trabajo y Promoción del Empleo, Instituto Nacional de Estadística e Informática (INEI), entre otros (las cuales al ser información brindada de manera oficial por estas entidades, le otorgan confiabilidad para su posterior uso). Entre la información recopilada se tiene:

- Serie histórica de la recaudación de tributos internos en la región Tacna (enero 2005-agosto 2020), incluida en el Anexo 01.

- Serie histórica anual de la Producción Bruto Interno de la región Tacna (2005-2016), incluida en el Anexo 02.

- Serie histórica anual de la tasa de desempleo de la región Tacna (2001-2018), incluida en el Anexo 03.

\section{Manejo y procesamiento de los datos}

Su disposición permite su manejo y sistematización, recurriendo para ello al uso de modelos econométricos estructurados sobre lo planteado en la teoría económica.

Dado que no se tiene una serie histórica comparable, se formuló el ajuste respectivo contemplando para ello los siguientes criterios:

Para cuantificar la producción bruta interna (PBI) de Tacna, se usó la variable recaudación de tributos internos (TI) en la región, la cual es una proporción "cuasi fija" de esta. Sin embargo, dado que los datos tributarios se encuentran expresados en términos nominales, resultó necesario deflactar el valor (usando el valor del IPC de Lima y Callao (ver Anexo 04), dada la ausencia de una serie histórica similar para la región Tacna) y uniformizar su valor base al definido para el PBI, para la definición del modelo, estructurado usando el método de mínimos cuadrados ordinarios (MCO)

$$
\widehat{T I}_{t}=\hat{\alpha}_{0}+\widehat{\alpha}_{1} P B I_{t}
$$

Donde:

$\widehat{T I}_{t}$ : Tributos internos recaudados en la región Tacna en el período $t$

$P B I_{t}$ : Producto Bruto Interno en la región Tacna en el período $t$

$\hat{\alpha}_{0}, \hat{\alpha}_{1}$ : Estimadores lineales del modelo.

Es así que en base a la proyección del volumen recaudado por impuestos internos para el presente año (2020), puede formularse una previsión del PBI local así como su variación anual.

Ya determinado el valor de la producción $(\mathrm{Y})$, se recurrió al uso de la denominada Ley de Okun, que permite relacionar a dicha variable con la tasa de desempleo (u), para tal fin se utiliza la siguiente expresión:

Donde:

$$
\frac{\Delta Y}{Y}=k-c \Delta u
$$

$\frac{\Delta Y}{Y}:$ Crecimiento de la producción entre períodos

$k$ : Porcentaje anual de crecimiento de la producción de pleno empleo

$c:$ Factor que relaciona los cambios en el desempleo con los cambios en la producción

$\Delta u$ : Cambio en la tasa de desempleo real entre períodos

\section{RESULTADOS}

Un primer acercamiento cuantitativo a los impactos analizados es el brindado por el análisis de la recaudación de tributos internos reportados en la región Tacna, con los que podemos determinar que el volumen captado entre los meses de enero a agosto en el año 2020 asciende a 107.1 millones de soles; lo que determina una variación de $-22.98 \%$ en relación al mismo período del año 2019 , en cuyo caso la recaudación fue de 139.1 millones de soles (Superintendencia Nacional de Administración Tributaria, 2020). Un factor que resulta conveniente incorporar 
en el análisis es el conjunto de facilidades que el Estado ha planteado para el pago de tributos e impuestos (como la reprogramación de los plazos de pago del Impuesto a la Renta), lo que definitivamente impacta en torno a un menor volumen captado.

Como puede apreciarse en la Figura 4, en el mes de mayo del presente año, el comportamiento de esta variable reflejó un mayor impacto, definiendo una contracción de $28.14 \%$ en referencia al mismo mes del año previo. Podemos notar adicionalmente una tendencia declinante ya desde inicio del año, la cual era también reflejo de situaciones externas que ya afectaban a la economía tacneña; entre estos elementos podemos destacar: el desarrollo de conflictos y protestas sociales en Chile, los cuales iniciaron en el mes de octubre del 2019 y que llevaron a que entre el 20 y el 26 de dicho mes se implementara el toque de queda en todo el país y se decretara el cierre de fronteras, retrayendo significativamente los arribos de los habituales visitantes chilenos a Tacna, y que pudiera explicar la menor recaudación de tributos internos en ese mismo mes (-6.71 \% menor al registrado en octubre del 2019).

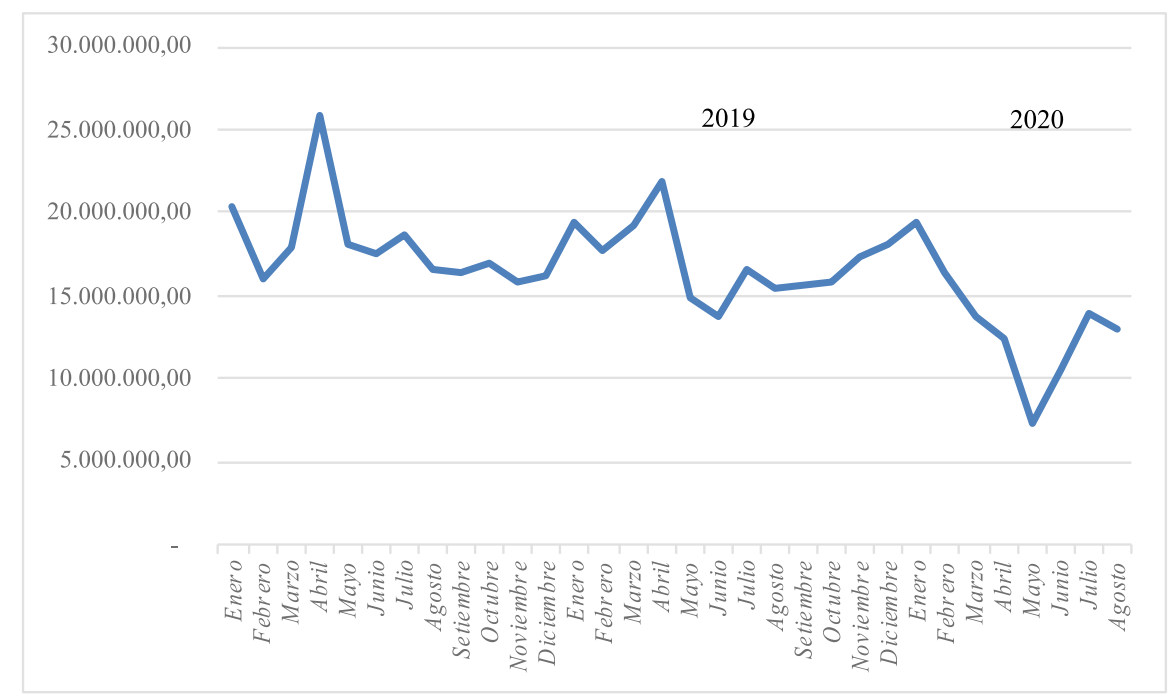

Figura 4. Región Tacna: Recaudación de tributos internos (enero 2018-agosto 2020)

Fuente: Superintendencia Nacional de Administración Tributaria (2020)

Si contemplamos el uso del registro de los datos que existen desde enero del 2005 y contemplando la conversión de estos valores a precios constantes del 2007 (en base al uso del valor del Índice de Precios al Consumidor o IPC de Lima y Callao para dicho período), encontramos un proceso de estancamiento de lo recaudado (ver Figura 5), lo cual podría implicar similar comportamiento de la producción agregada de la región. Es así que los tributos internos captados en el año 2020 son equivalentes a los obtenidos hace tres lustros, es decir, los recursos captados durante el año 2005.

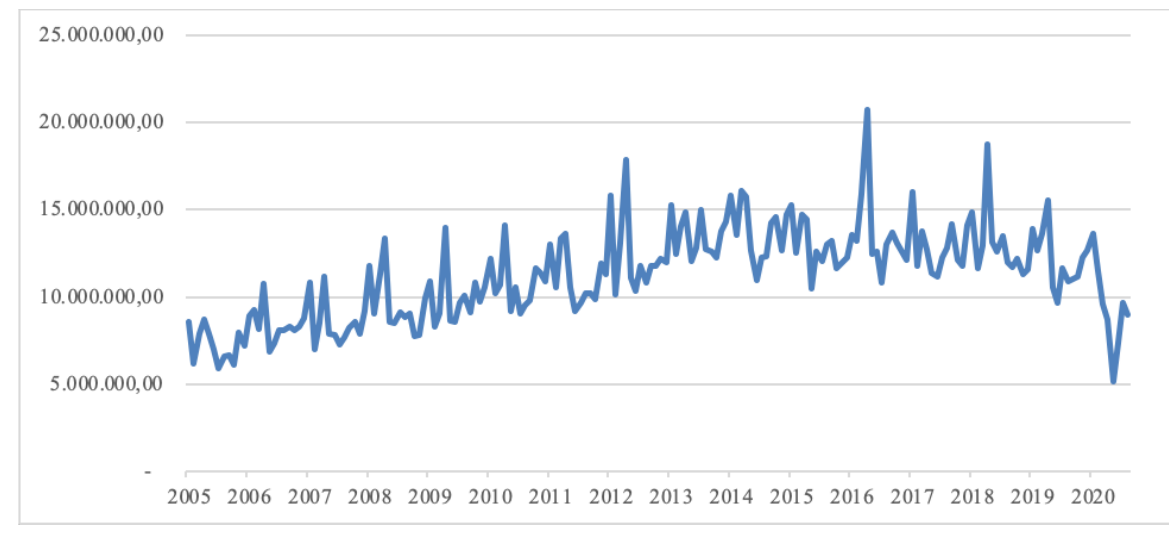

Figura 5: Región Tacna: Recaudación de tributos internos a precios constante en soles del 2007 (enero 2005 - agosto 2020)

Fuente: Superintendencia Nacional de Administración Tributaria (2020) 
Para validar lo planteado previamente, analizamos la evolución del PBI regional en un período más o menos similar (2005-2016), en el cual y como se aprecia en la Figura 6, la economía de Tacna creció a una tasa promedio anual de $2.91 \%$, cifra apenas superior al valor de $1.96 \%$ definida como la tasa promedio de crecimiento intercensal; lo cual, nos plantea el escaso crecimiento real en este espacio territorial (Instituto Nacional de Estadística e Informática, 2020).

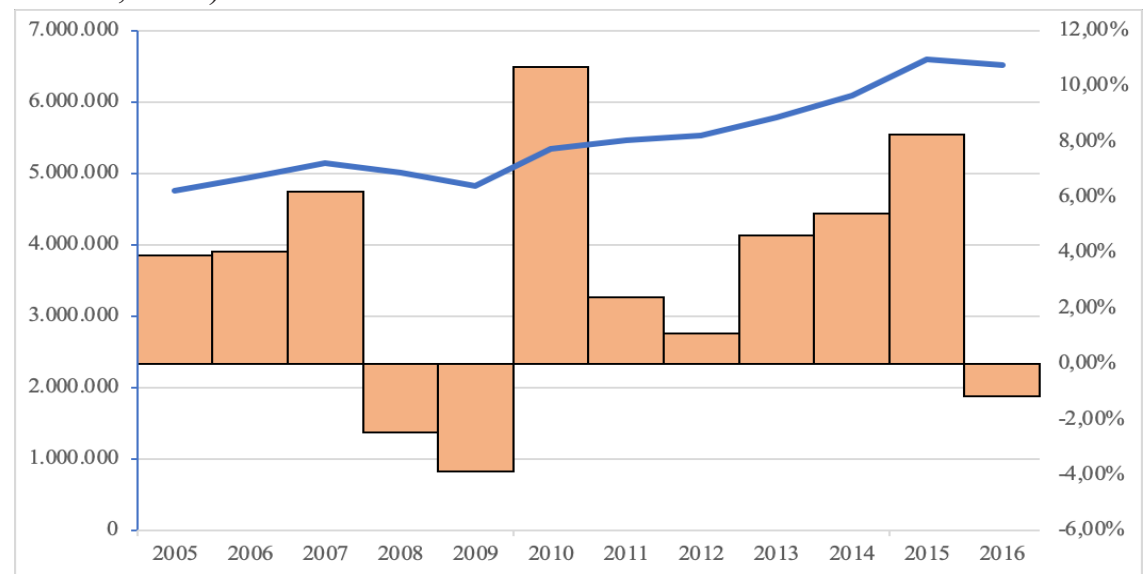

Figura 6. Región Tacna: PBI a valores constantes, en miles de soles del 2017 y tasa de variación anual (2005-2016)

Fuente: Instituto Nacional de Estadística e Informática (2020)

La vinculación entre las variables puede verse reflejada al construir el siguiente modelo que explica la recaudación en base al PBI (para el período 2005-2016):

$\widehat{T I}_{t}=-5676,9+0,3501 \cdot P B I$

La cual aprecia una bondad de ajuste de $R^{2}=0,9615$; y configura una recaudación marginal del orden del 35.01 $\%$ respecto a la producción incremental regional.

Proyectando el valor de la producción agregada para los años 2017 al 2019, en base a la data de ambas variables, se obtienen los valores de 454.5, 460.3 y 431.1 miles de soles del 2017. Adicionalmente y considerando la tributación interna (expresada también en términos constantes en soles del 2017) recaudada hasta agosto del 2020, puede denotarse una reducción del $24.21 \%$ en referencia al año 2019. Si esta tendencia se mantiene hasta el final del año podría preverse una variación de $-22.44 \%$ en el PBI para el presente año (2020).

En cuanto al desempleo (Figura 7), desde el 2003 (en que se suscitó una tasa de $7.3 \%$ ) se observa una tendencia decreciente hasta alcanzar casi una condición de pleno empleo en el 2018 con un registro de $2.8 \%$ (Ministerio de trabajo y promoción del empleo, 2019).

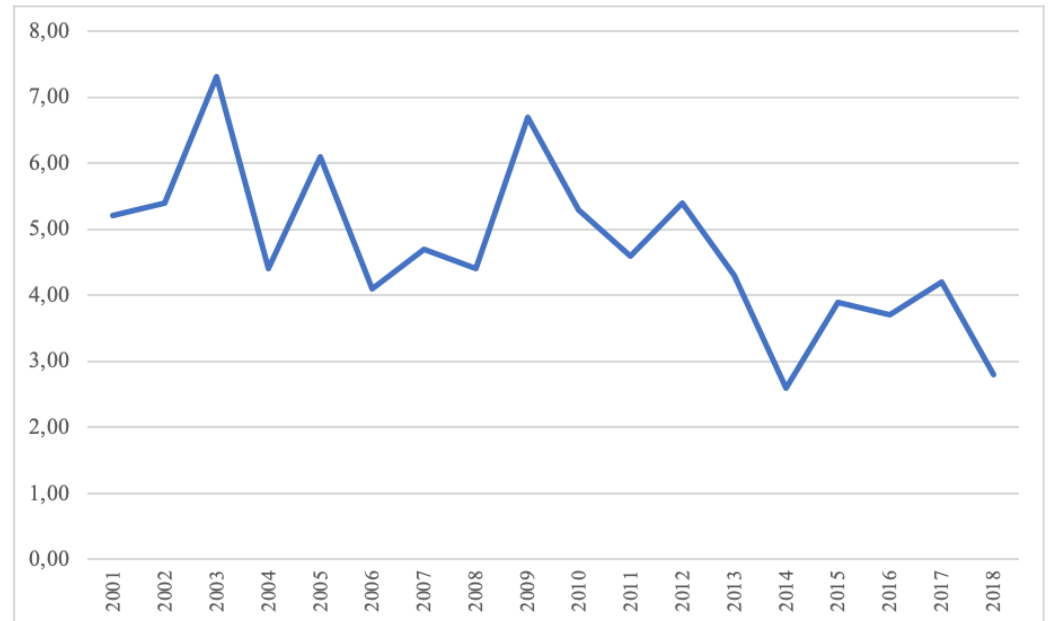

Figura 7. Región Tacna: Tasa de desempleo anual, en porcentaje (2001-2018)

Fuente: Ministerio de trabajo y promoción del empleo (2019) 
Considerando la información del año 2017, subsiste un problema significativo en el mercado laboral de Tacna; y es que solo un $57.1 \%$ de la población económicamente activa (PEA) se encuentra adecuadamente empleada y un $38.7 \%$ exhibe una condición de subempleo $(3.1 \%$ con un subempleo por horas y $35.6 \%$ con subempleo por ingresos), lo que describe una alta volatilidad y precariedad en la renta obtenida por la PEA regional (Ministerio de trabajo y promoción del empleo, 2019).

Un aspecto adicional por considerar es la distribución del empleo por sectores, lo cual nos permite determinar que un $70.4 \%$ de la PEA se encuentra orientada a los sectores llamados "terciarios" o de prestación de servicios, sector que solamente aporta con un $26.19 \%$ al PBI; esto implica una alta concentración del mercado laboral en una actividad saturada y probablemente poco dinámica.

Evidentemente, existe una relación entre la producción generada y la tasa de desempleo, así y en base de los datos para el período 2002-2018 es factible construir la ecuación descrita por Okun:

$$
\frac{\Delta Y}{Y}=3,1656-0,6012 \Delta u
$$

Esto nos permite definir una tasa de crecimiento anual en pleno empleo de $3.17 \%$ en el PBI, un factor de 0.60 para la relación entre los cambios en el desempleo con los cambios en la producción y un coeficiente de Okun de 0.1183 .

Si la economía de Tacna medida a través de su PBI se contrae en $22.44 \%$ el presente año (2020); y en base a la ecuación planteada en atención a la ley de Okun, podríamos determinar un incremento en la tasa de desempleo en el 2020 del orden de $32.06 \%$. Esto implica que casi una tercera parte de la población ocupada engrose el grupo de desempleados, lo cual plantea un indicador preocupante y un reto en la construcción de políticas públicas orientadas a propiciar el beneficio social de la comunidad.

La recuperación no será un proceso sencillo porque implicará la reestructuración del sistema económico, basado en la identificación de las vulnerabilidades ya evidenciadas, al menos si se desea plantear sustentabilidad al desarrollo de la región Tacna.

De mantenerse un deterioro en las condiciones económicas, esto no solo impacta en los resultados o indicadores comúnmente poco escrutados de manera pública, su mayor efecto se plantea a nivel del tejido social de la región y cuya descomposición es más difícil de enfrentar.

\section{DISCUSIÓN}

La presencia del COVID-19 en definitiva ha generado impactos negativos importantes en la economía mundial; así The World Bank (2020) prevé una contracción de $5.2 \%$ en la producción del planeta para el presente año, configurando una de las mayores recesiones que el mundo ha enfrentado en las últimas décadas. El mismo informe plantea que la región más golpeada será definitivamente América Latina con una reducción de $-7.2 \%$.

Las economías más sensibles son indudablemente aquellas que observan mayores niveles de marginalidad, aspecto común en las distintas regiones que conforman nuestro país. Esto es delineado por Gonzales, et. al. (2020) al plantear que:

"La COVID-19 y la implementación de la estrategia de supresión han generado shocks de oferta y demanda agregada, con los consiguientes costos económicos y sociales: insolvencia de empresas, aumento del desempleo, mora bancaria, disminución de ingresos laborales, reducción del consumo y ahorro, además de la postergación de las decisiones de inversión, lo que implicaría una recesión a futuro."

De igual modo, Jiménez (2009) propone que las crisis internacionales tienen efectos recesivos y fiscales significativos en nuestro país, por la insuficiencia de la demanda interna y la alta dependencia que se tiene respecto a las importaciones y la demanda externa.

Uno de los primeros elementos que determinó un impacto negativo en la economía fue la caída inicial de los precios de las materias primas en general, y en particular de los minerales (considerando que es la actividad más 
importante de la región), esto es reseñado y visibilizado por Jaramillo y Ñopo (2020), así como la gravitante informalidad, lo que los lleva a estimar impactos significativos en la producción para el presente año.

No obstante, los reportes en la evolución de los precios internacionales desde abril y mayo exhiben una tendencia positiva, a partir de la mejora en la presencia de casos en los principales demandantes de bienes y que pudieran propiciar mitigar mucho de los impactos previstos inicialmente (Banco Central de Reserva del Perú, 2020).

En contraparte, el mantenimiento de los cierres fronterizos en toda la región abona significativamente en una mirada no positiva para la evolución del PBI regional, y es que un $60 \%$ de los turistas extranjeros que visitan Tacna residen en Chile, generando un gasto per cápita de US\$230 por visita (PROMPERU, 2017).

Finalmente, en torno a la actividad minera que básicamente se encuentra explicada por la producción de cobre en Toquepala, esta no ha sufrido mayores impactos debido a la no paralización de sus labores durante la implementación de las medidas de confinamiento que sí afectaron a otras actividades productivas, al focalizar su área productora en espacios alejados de los principales centros urbanos de la región. Esto permitirá además mantener (ya pensando en el 2021) en cierto modo la disposición de recursos de gasto público originado por los rubros de canon, sobrecanon y regalías mineras para las instancias de gobiernos subnacionales; a pesar de observar cómo se destacó una contracción de los tributos internos durante el presente año lo que implicará en la reducción de los recursos denominados como ordinarios, además de aquellos captados por los servicios brindados por estas instituciones.

Una adecuada orientación de estos recursos en actividades con alto efecto multiplicador aportaría a la tarea de reducir los impactos en la producción y fundamentalmente el desempleo, marcando pautas de la agenda pública en los próximos meses.

Por otro lado, el reinicio de algunas actividades productivas incorporadas en las denominadas fases 3 y 4 de la reactivación económica prevista por el gobierno pudieran dinamizar en cierto modo algunos negocios y emprendimientos; no obstante, muchas organizaciones y empresas probablemente no retornen al haber colapsado ante la imposibilidad de afrontar un período demasiado extenso sin registrar mayores ingresos.

Ya en torno al impacto en el empleo, la Organización Internacional de Trabajo (2020) explora en las repercusiones de la cantidad y calidad del trabajo, pero adicionalmente sobre efectos específicos sobre grupos de mayor vulnerabilidad. Asimismo, estima un aumento del desempleo mundial de entre 5.3 millones (hipótesis "prudente") y 24.7 millones (hipótesis “extrema”) en este 2020.

Un estudio formulado por Banco Central de Chile (2020) plantea que Perú y Colombia serán los países en Sudamérica con mayores impactos en el empleo, motivados por la presencia de la pandemia.

El planteamiento formulado en la investigación sobre el análisis del desempleo a partir de la evolución de la producción es revisado por Gonzalez (1999), quién demuestra que la ley de Okun se aplica a países como el nuestro, notando también la mayor volatilidad en la respuesta respecto a otros con mayor nivel de formalidad como es EE.UU.

Cabe precisar que, Garavito (2002) determina un coeficiente de Okun igual a 0.0845 para nuestro país, durante el período 1970-2000; el cual es ligeramente inferior al obtenido en nuestro modelo y semejante al generado por el trabajo formulado por Laos (2014) que estima un coeficiente de 0.1345 .

Pensar en un crecimiento del desempleo del $22.44 \%$ supone un reto importante pues determina una quinta parte de la PEA sin la disposición de trabajo y con ello la reducción de sus ingresos habituales, lo cual a la par impactará en un menor volumen de consumo, lo que implicará una mayor contracción de la demanda interna y una mayor dependencia de la demanda externa, también fuertemente golpeada por la pandemia.

\section{CONCLUSIONES}

La presencia del virus COVID-19 ha generado una emergencia sanitaria que ha motivado una serie de medidas, la mayor parte de ellas restrictivas a fin de minimizar la circulación y tránsito de personas con la finalidad de minimizar la presencia de la enfermedad en el mundo, aspecto del que no se escapa nuestro país y la región. 
La implementación de las medidas ha tenido y viene teniendo enormes impactos en la actividad cotidiana de las personas, la cual se hace más significativa para economías como la nuestra, orientadas a las actividades terciarias (más de 2/3 parte de la PEA orientada hacia ellas) con precariedad e informalidad.

Uno de los primeros aspectos resaltantes es la notoria contracción que se ha evidenciado en la recaudación de tributos internos. Los datos presentados nos permiten mostrar que dicha variable se ha reducido en $22.98 \%$ (pero $24.21 \%$ en términos reales) para el período comprendido entre enero y agosto del presente año y en relación a lo exhibido el 2019.

Una menor recaudación tributaria es un claro efecto de una menor producción, tanto de bienes como servicios; motivado por la reducción de la demanda interna (ya golpeada por el confinamiento y sus efectos), pero también por la ausencia de la habitual demanda externa que impacta en el sector que absorbe a la mayor cantidad de población empleada. Por ello, presumir una variación de - 22.44 \% para el PBI del presente año (2020) no resulta descabellado y nos permite establecer lo profundo del estado recesivo de la región Tacna.

Basados en una menor producción y considerando los resultados que nos ofrece la determinación de un modelo estructurado en base de la denominada ley de Okun, puede predecirse un incremento de $32.06 \%$ en la tasa de desempleo regional en el 2020.

Resulta fundamental prospectar y generar una mirada que permita establecer medidas orientadas a enfrentar este período recesivo y evitar que este tenga una duración extensa, debido a los impactos no solo económicos que podría implicar. Esto debe además conjugarse con un nuevo escenario donde la preocupación por el estado de la pandemia aun no puede perderse de vista, más aún si la disposición de una vacuna y su implementación no serán inmediatos.

\section{REFERENCIAS}

Banco Central de Chile. (2020). El impacto del COVID-19 en el mercado laboral. Santiago: Banco Central de Chile.

Banco Central de Reserva del Perú. (2020). Resumen Informativo Semanal del 24 de setiembre del 2020. Lima: BCRP.

Contreras, Y., Tapia, M., \& Liberona, N. (2017). Movilidades y prácticas sociales fronterizas entre Tacna y Arica. Del sentido de la frontera a la transfronterizidad entre ciudades. Diálogo andino, 127-141.

Dilla, H., \& Álvarez, C. (2018). Arica/Tacna: Los circuitos económicos de un complejo urbano transfronterizo. Diálogo andino, 99-109.

Dirección Regional de Salud de Tacna. (29 de Setiembre de 2020). DIRESA Tacna. Obtenido de Situación actual COVID-19: https:/www.facebook.com/drstacna/photos/a.1550355185270233/2416891728616570/

Garavito, C. (2002). La ley de Okun en el Perú: 1970-2000. Lima: PUCP.

Gonzalez, J. (1999). Labor market flexibility in thirteen Latin American countries and the United States. Stanford: World Bank Books.

Gonzales, J., Varona, L., Domínguez, M., \& Ocaña, V. (2020). Pandemia de la coviD-19 y las Políticas de Salud Pública en el Perú: marzo-mayo 2020. Revista de Salud Pública, 1-9.

Google Inc. (29 de Setiembre de 2020). Informes de movilidad de las comunidades ante el COVID-19. Obtenido de Informes de movilidad de las comunidades ante el COVID-19: https://www.google.com/covid19/mobility/?hl=es-419

Instituto Nacional de Estadística e Informática. (29 de Setiembre de 2020). INEI. Obtenido de Estadísticas: https://www.inei.gob.pe/estadisticas/indice-tematico/producto-bruto-interno-por-departamentos-9089/

Jaramillo, M., \& Ñopo, H. (2020). Covid-19y el shock externo: Impactos económicos y opciones de política en el Perú. Nueva York: PNUD América Latina y el Caribe.

Jiménez, F. (2009). Perú: Crisis y restricciones al crecimiento. EconoQuantum, 143+.

Laos, R. (2014). Relación entre crecimiento económico y tasa de desempleo en el Perú, 2000-2013. Trujillo: Universidad Nacional de Trujillo.

Ministerio de trabajo y promoción del empleo de Tacna. (2019). Región Tacna: panorama laboral. Lima: Dirección de investigación socio económico laboral. 
Organización Internacional de Trabajo. (2020). El COVID-19 y el mundo del trabajo: Repercusiones y respuestas. Ginebra: OIT.

PROMPERU. (2017). Perfil del Turista Extranjero 2016. Lima: PROMPERU.

Sistema Informático Nacional de Defunciones. (29 de Setiembre de 2020). SINADEF. Obtenido de Tablero de control: https://www.minsa.gob.pe/reunis/data/defunciones_registradas.asp

Superintendencia Nacional de Administración Tributaria. (29 de Setiembre de 2020). SUNAT. Obtenido de Nota tributaria y aduanera: http://www.sunat.gob.pe/estadisticasestudios/ingresos-recaudados.html

The World Bank. (2020). Global Economic Prospects. Washington D.C.: World Bank Group. 